\title{
RELACIONES ENTRE LA GEOMORFOLOGÍA Y LA HISTORIA: EL ESTE Y NORESTE DE ESTADOS UNIDOS DE NORTEAMÉRICA
}

Por. Ing. Dr. Eliseo Popolizio

Diagramación y compaginación: Ing. Eduardo Barrios

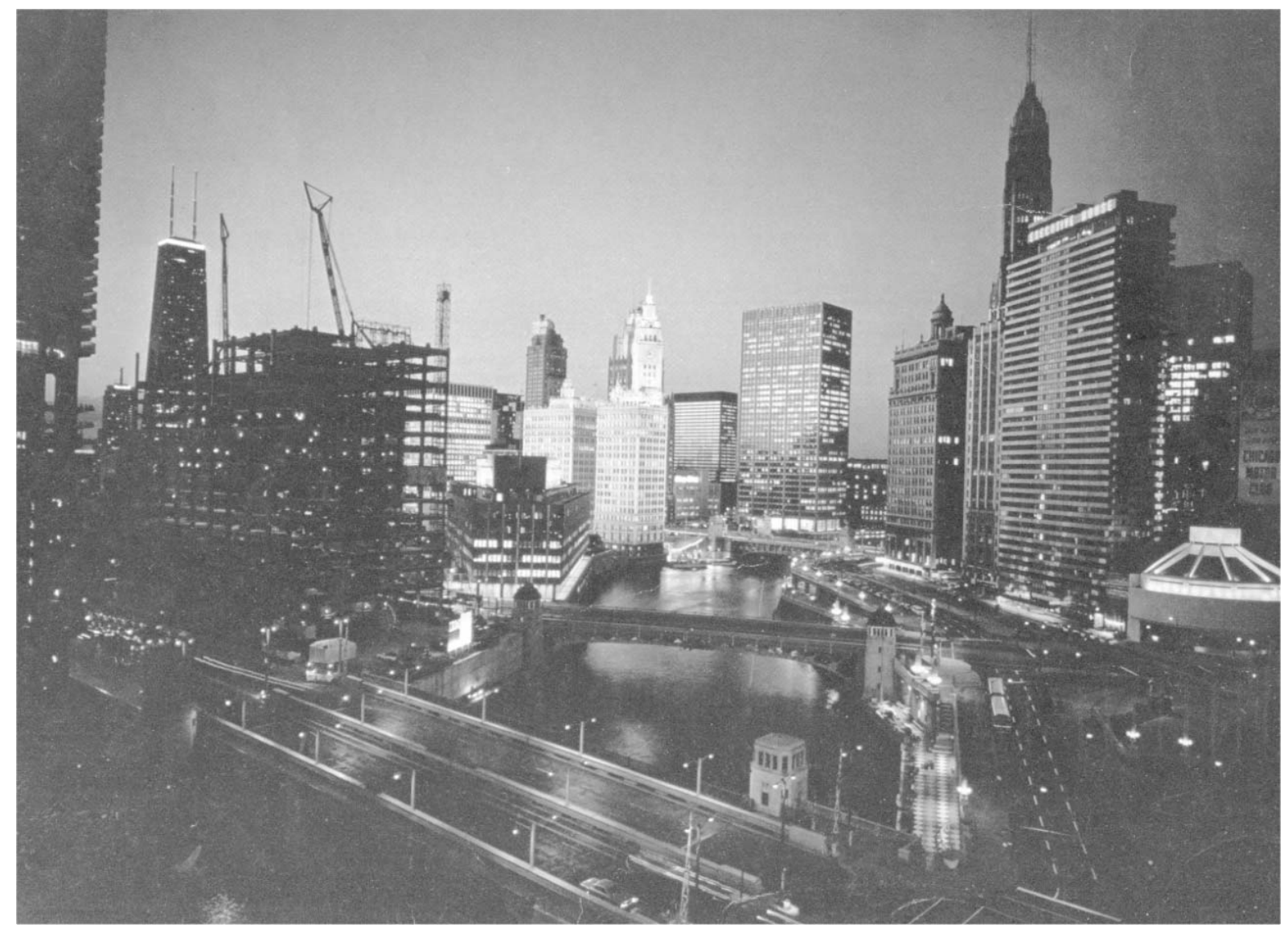




\title{
Por. Ing. Dr. Eliseo Popolizio
}

Centro de Geociencias Aplicadas. UNNE. Las Heras 727. Resistencia.

e-mail: epopolizio@ing.unne.edu.ar.

\section{Resumen}

Entender la organización del espacio en el este y noroeste de los Estados Unidos de Norteamérica, requiere tener en consideración los aspectos geomorfológicos que condicionaron las actividades, la localización de los centros urbanos y los desplazamientos en la progresiva ocupación del territorio.

Las favorables condiciones morfológicas y geológicas relacionadas con los yacimientos de carbón, hierro y petróleo y las vías fluviales y lacustres explican, en parte, el desarrollo y la prioridad de ciertos centros urbanos y de la megalópolis Waston.

El trabajo pretende retomar los tradicionales conceptos de la Geografía y mostrar la importancia del análisis geomorfológico en los estudios regionales.

Palabras claves: Geomorfología Aplicada, Estados Unidos de Norteamérica, Historia, Geografía Económica.

\section{RELATE BETWEEN THE GEOMORPHOLOGY AND THE HISTORY: THE EAST AND NORTHEAST OF TOGETHER STATES DE NORTH AMERICA}

\begin{abstract}
To understand the organization of the space in the east and northwest of the United States of North America, he/she requires to have in consideration the geomorphological aspects that conditioned the activities, the localization of the urban centers and the displacements in the progressive occupation of the territory.

The favorable morphological and geologic conditions related with the locations of coal, iron and petroleum and the waterways and lacustrine they explain, partly, the development and the priority of certain urban centers and of the megalopolis Waston.

The work seeks to recapture the traditional concepts of the Geography and to show the importance of the geomorphological analysis in the regional studies.
\end{abstract}

Key words: Applied geomorphology, United States of North America, History, Economic Geography.

\section{Desarrollo}

Este trabajo se propone considerar el condicionamiento entre la Geomorfología del Este de los Estados Unidos de Norteamérica y algunos acontecimientos históricos que influyeron en la organización espacial del territorio.

En la Fig 1 se esquematiza los principales rasgos del área en consideración, ampliamente estudiada por geólogos y geomorfólogos, destacándose: la Planicie Costera, Los Apalaches y el área de Los Grandes Lagos

La llegada de los europeos al continente se produjo en la primera de esas unidades geomorfológicas y en ella una línea de falla la recorre todo a lo largo, originando un desnivel y saltos y correderas en los cursos fluviales. 


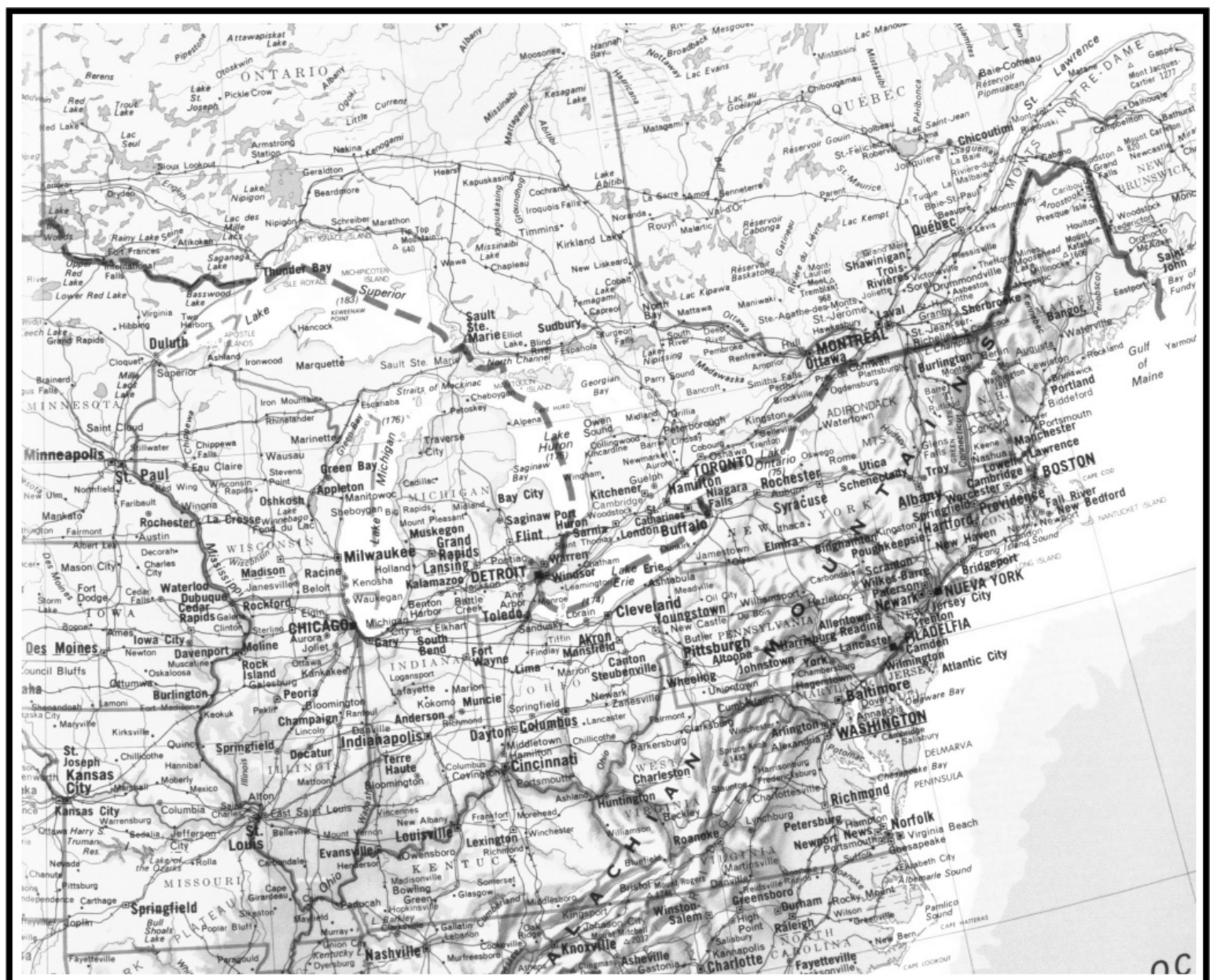

Fig. 1: Mapa del sector noreste de los Estados Unidos donde se distinguen la planicie costera, los Apalaches, la meseta apalacheana y los grandes lagos.

Los primeros asentamientos se originaron en la costa como pequeños núcleos aislados dando lugar a las 13 colonias iniciales y desde allí se inició la penetración hacia el oeste por los cursos fluviales, donde fue necesario trasbordar las mercancías transportadas en barcazas de fondo plano, dando lugar a pequeños puestos permanentes, que paulatinamente fueron creciendo y fueron el origen de ciudades tales como: Nueva York, Filadelfia, Washington, etc., a lo largo de la línea de falla. (Fig. 2). Inicialmente la disponibilidad de energía hidráulica, por el resalto hidrológico, permitió la instalación de molinos harineros a paletas movidas por el 


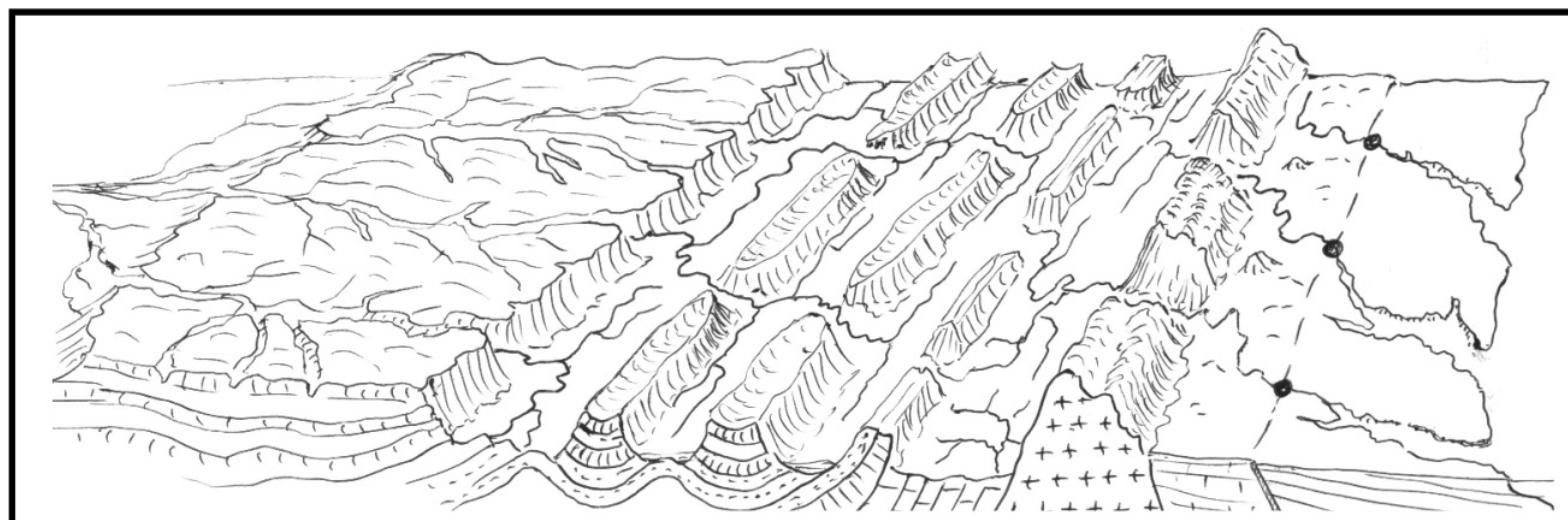

Fig. 2: Modelo esquemático indicando de izquierda a derecha: la meseta apalacheana, los Apalaches, los montes Azules, la planicie costera y la línea de falla, donde se instalaron los primeros núcleos vinculados al transbordo de mercaderías.

agua y posteriormente el desarrollo de otras industrias, de manera que se fueron creando núcleos urbanos. 


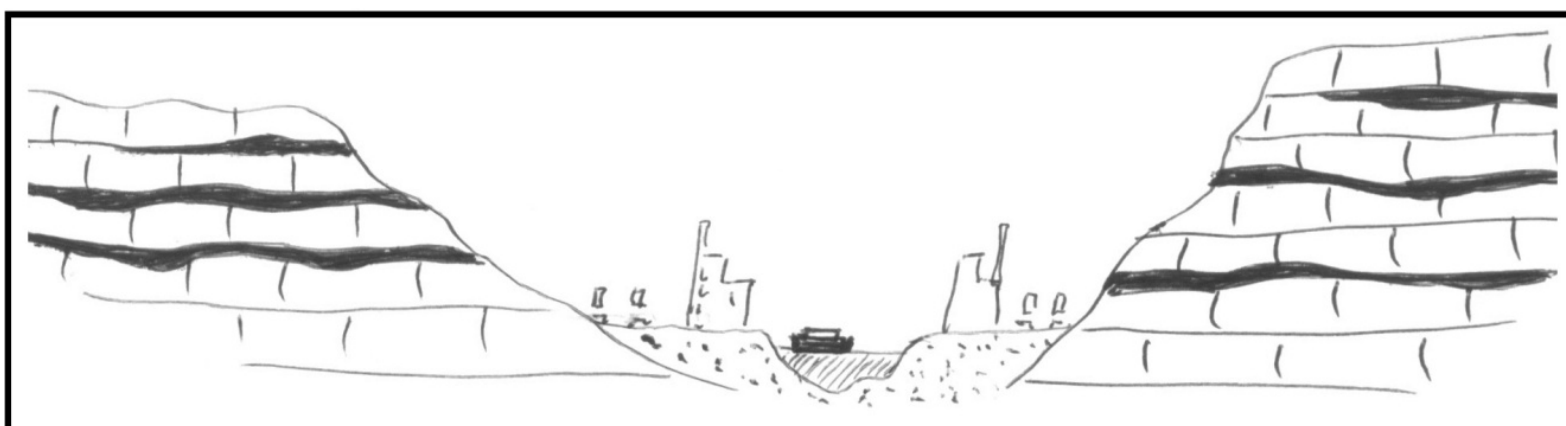

Fig. 3: Corte esquemático de los valles fluviales que atraviesan la meseta Apalacheana, donde se puede observar la disposición, casi horizontal, de los estratos de carbón y la facilidad de su transporte por via terrestre o fluvial.

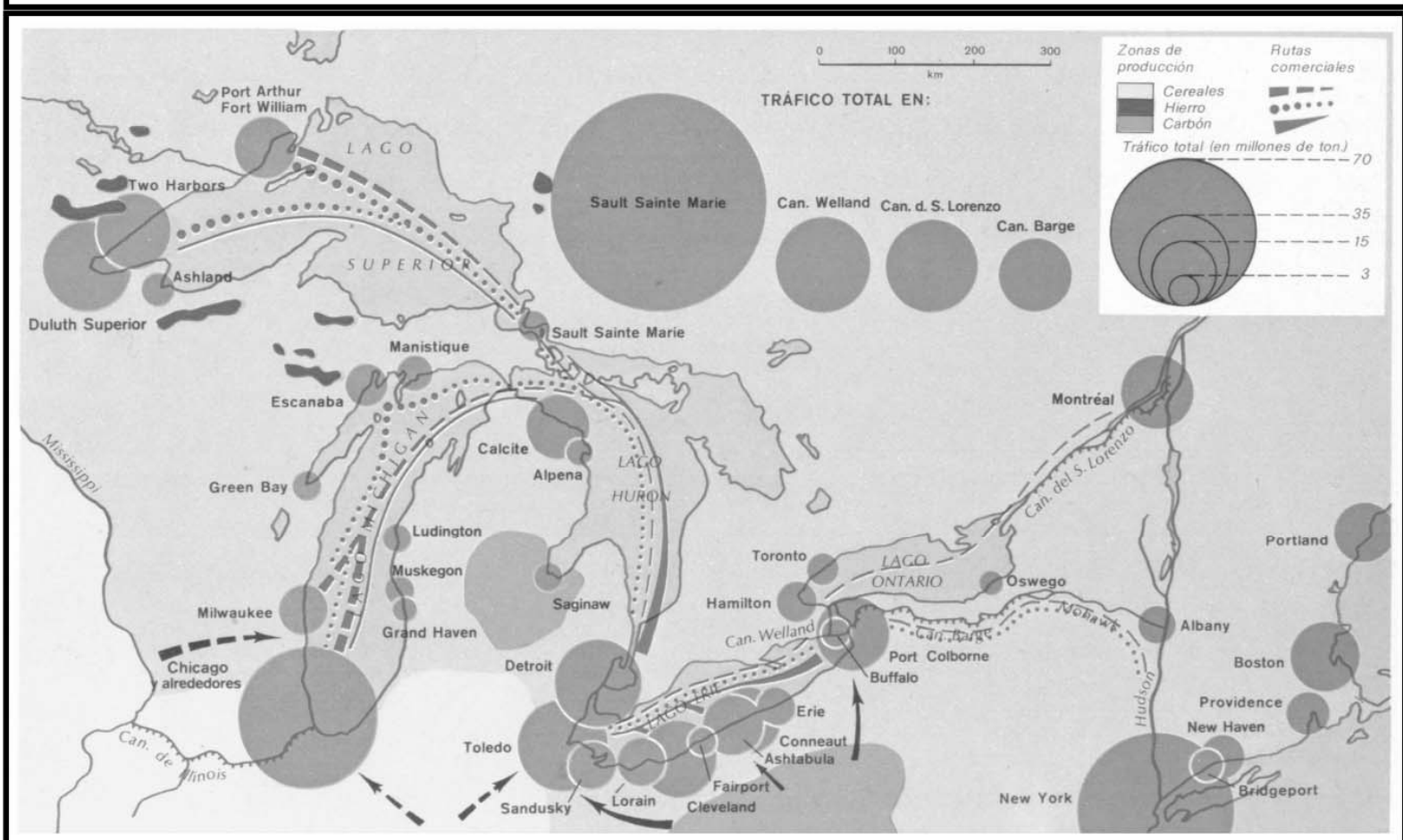

Fig. 4: LA REGIÓN DE LOS GRANDES LAGOS. Circundados por regiones de elevada producción agraria, minera e industrial, los grandes lagos constituyen una importante vía de circulación. con un considerable tonelaje de mercancías transportadas anualmente, pese a que la navegación se interrumpe en los meses invernales. Los círculos correspondientes a los diversos puertos indican el tráfico local, mientras que los cuatro círculos trazados en la parte superior del mapa permiten comparar el movimiento total en los cuatro puntos clave situados entre los grandes lagos. La circulación por el canal de San Lorenzo está destinada a incrementarse en el futuro.

Cuando la base energética de la industrialización era el carbón, los americanos había atravesado los Apalaches y allí encontraron estratos muy ricos en este mineral, de manera que fue posible el rápido desarrollo de las industrias algodoneras y la concentración de la población en los núcleos primitivos.

Una vez atravesado el relieve montañoso los americanos llegaron a la Meseta Apalacheana, donde los estratos carbonosos están casi horizontales y aflorando a lo largo de los cursos fluviales, a diferencia de Inglaterra donde se hunden a gran profundidad y por lo tanto el costo de explotación fue mucho menor que en la Madre Patria. 


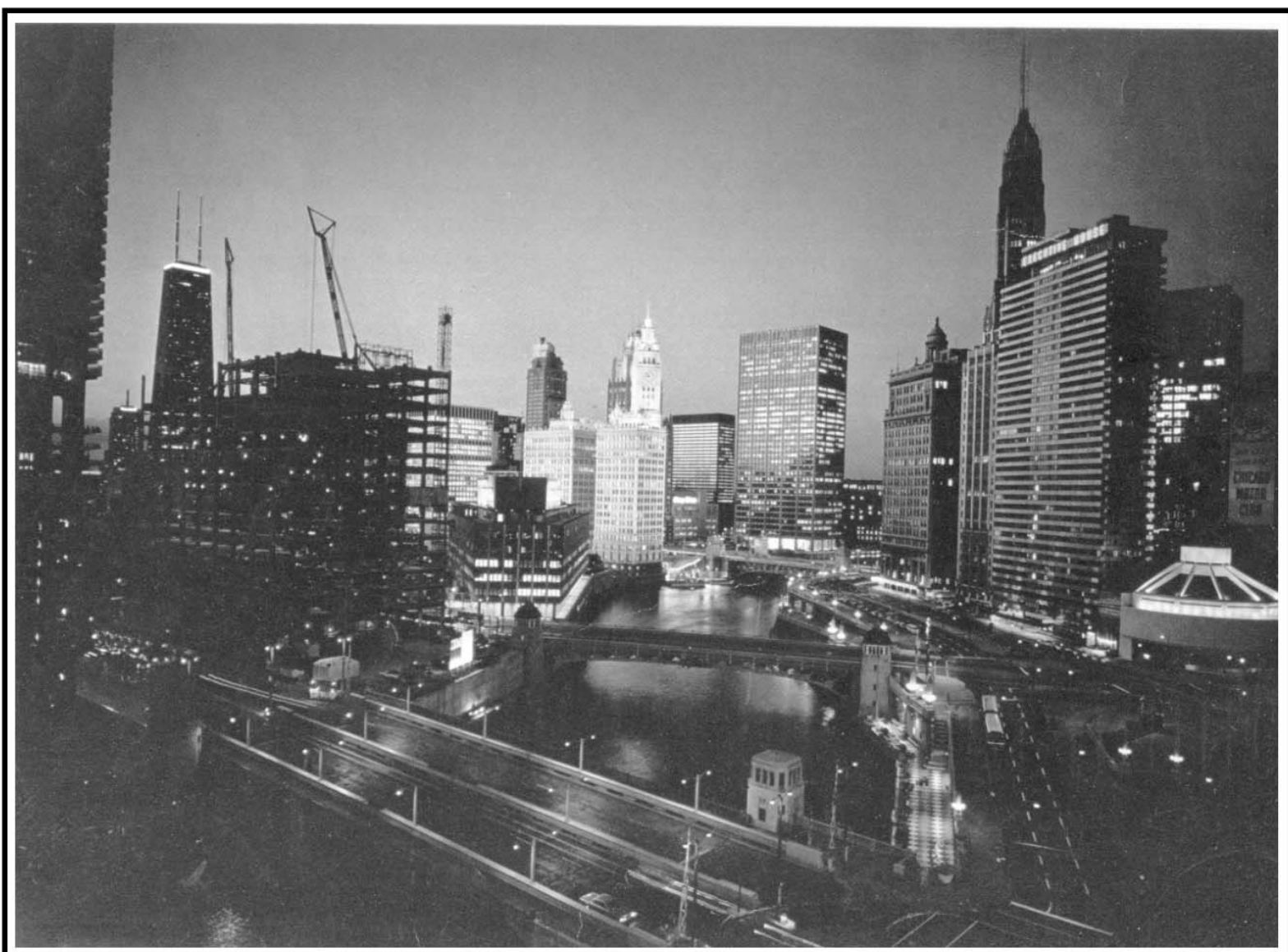

Fig. 5: Canal de navegación atravesando la ciudad de Chicago, uniendo el sistema de los grandes lagos con el del río Mississippi.

Como los cursos tenían terrazas amplias se podía circular fácilmente por los valles y en ellas se instalaron las primeras industrias y los depósitos del carbón extraído, que finalmente se transportaba a los ferrocarriles (Fig. 3).

Pero si observamos la red de cursos emplazados en la meseta veremos que convergen hacia un punto donde se desarrolló otro asentamiento humano: la ciudad de Pittsburgh, la cual creció rápidamente, como capital del carbón y sede de numerosas industrias.

Por otra parte en el sur del Lago Superior se descubrieron importantes yacimientos de hierro, cuyo procesamiento con el carbón permite fabricar acero y a mitad de camino entre ellos y Pittsburgh existía otro emplazamiento urbano en el extremos sur del Lago Michigan: Chicago (Fig. 4).

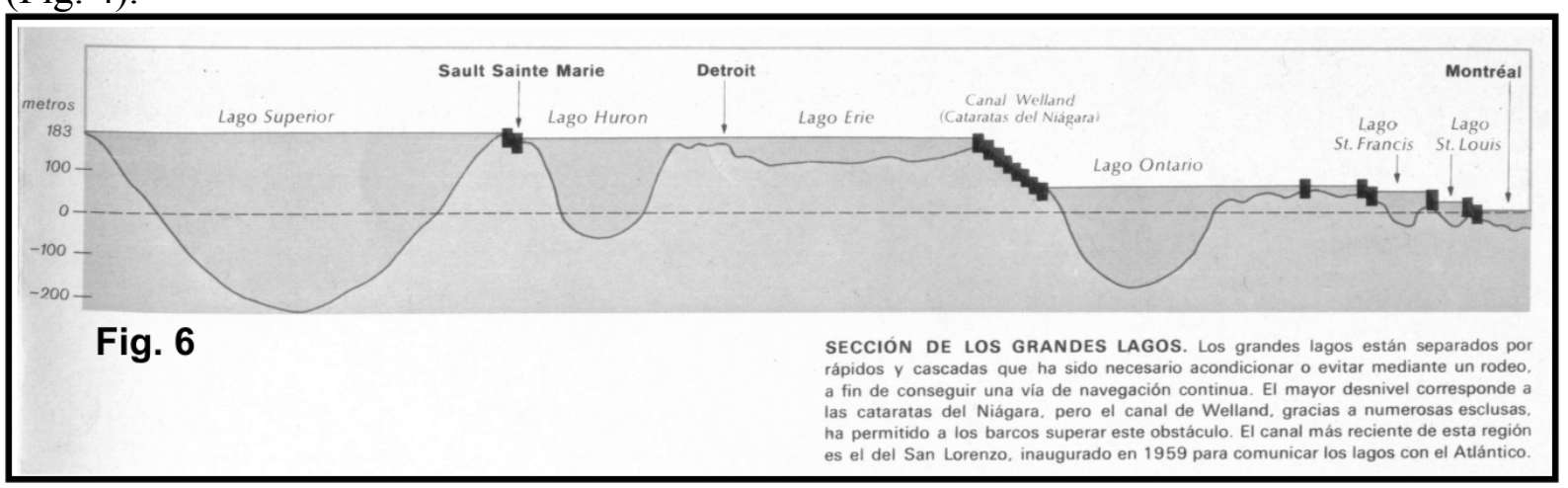

De esta manera esta última era el lugar estratégico para la fabricación de acero y se desarrolló allí la mas grande acería de Estados Unidos, la Steel Company. Pero, la ciudad estaba emplazada en el extremo norte de la gran zona maicera y ganadera del país, de manera que la producción de maquinaria agrícola fue una de las actividades básicas de la ciudad 


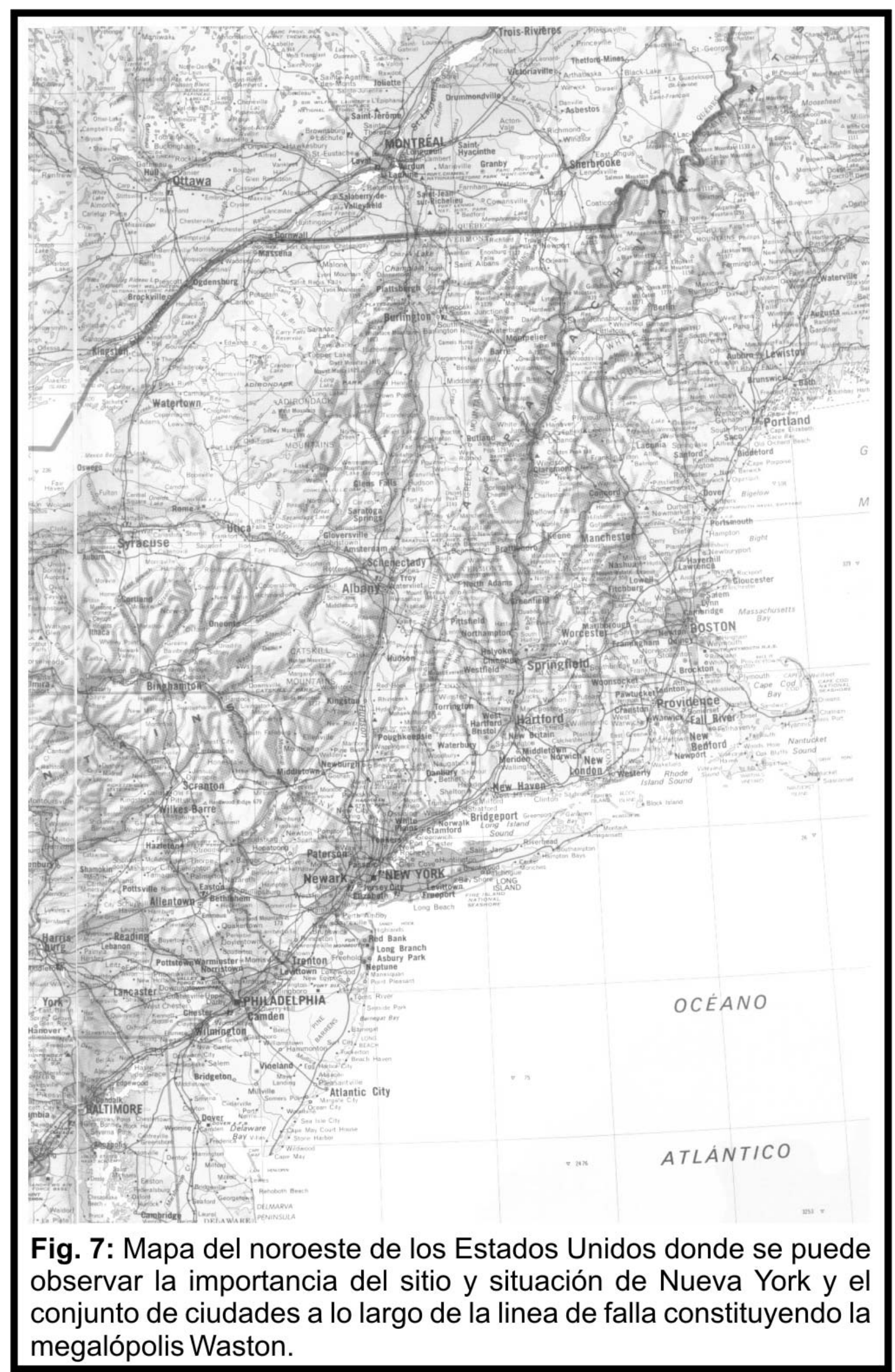


Además, los Grandes Lagos originan una zona microclimática a su alrededor, menos rigurosa y permite la formación de una faja de producción de frutales y el desarrollo del ganado lechero.

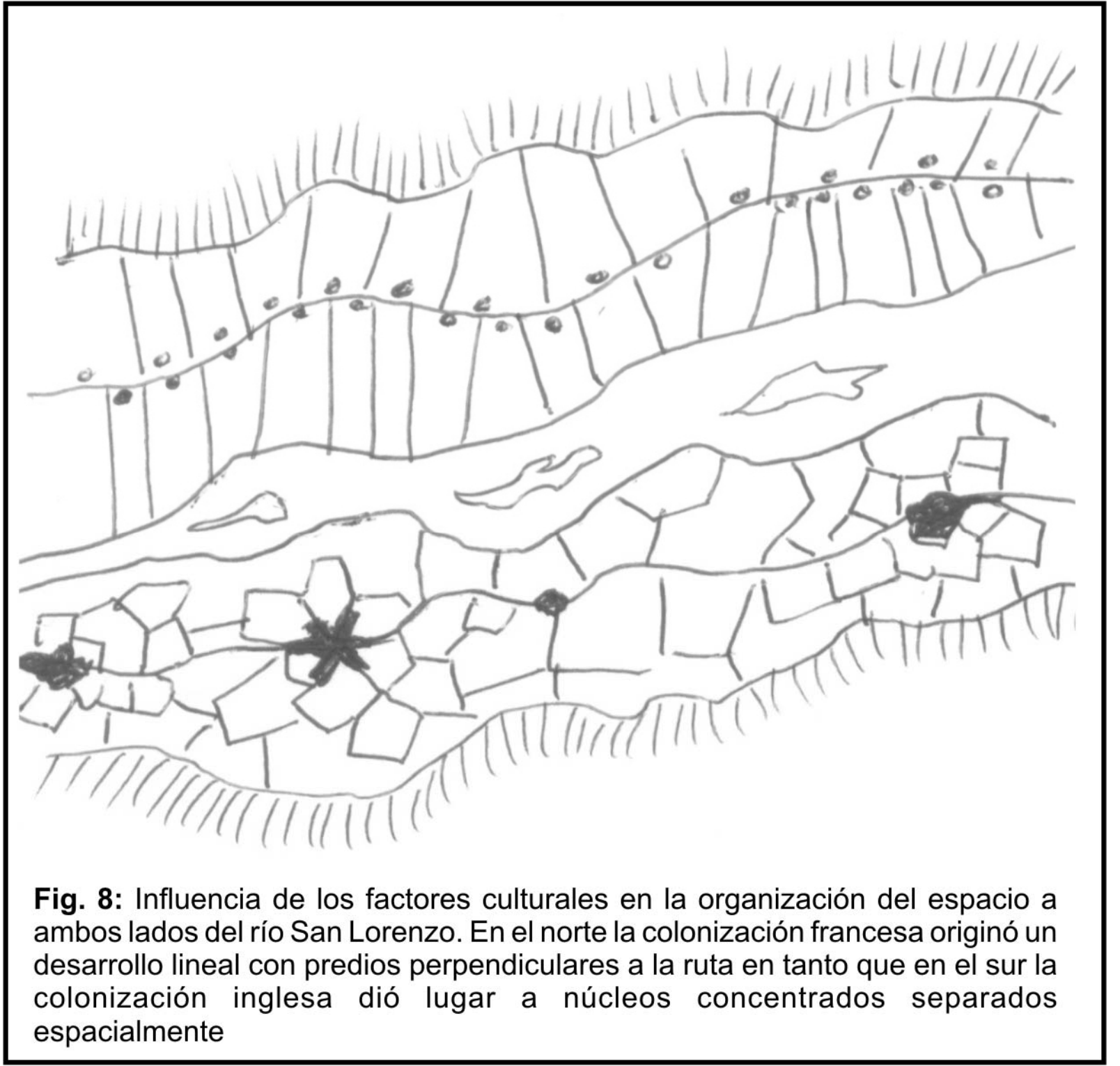

De manera que las condiciones naturales son una de las causas condicionantes del desarrollo en el Medio Oeste de la gran metrópoli de Chicago, a lo cual se sumaron otros resultantes de la combinación de estas con las obras humanas.

La proximidad del Lago Míchigan al sistema del río Mississippi permitió que se construyera un canal entre ambos que atraviesa la ciudad y permitió el transporte fluvial hacia el sur en dirección a Nueva Orleáns (Fig. 5).

La navegación hacia el este estaba dificultada por las cataratas del Niágara, pero en el siglo pasado una obra enorme de ingeniería logró vencerlas mediante un canal lateral que permite el paso de buques de ultramar, originando lo que se llamó El Octavo Mar (Fig. 6).

Chicago se convirtió de esta manera en un polo de desarrollo enorme y puerto fluviomarítimo que unía el Atlántico Norte con el Golfo de México y en el nudo de comunicaciones aéreas más importante

Pero, ¿Qué pasó con Nueva York? . No era la principal ciudad en los tiempos de la colonia, aventajada por Boston y Filadelfia y llegó a convertirse el la ciudad más grande de los Estados Unidos 
Su emplazamiento desde el punto de vista geomorfológico es estratégico, ya que se inicia en la isla de Manhatan, comprada a los Holandeses en la desembocadura del río Hudson, que recibe por el norte la Mohawk, de manera que ambos valles forman una especie de triángulo delimitando los montes Adirondack y a través de ellos se accede a los lagos y al río San Lorenzo (Fig. 7).

El valle del río Hudson es de origen glaciar, originado por una lengua de hielo que descendía del enorme casquete de hielo que cubría Canadá durante la última glaciación. Como resultado de ello, se originó un corredor natural hacia los lagos, con depósitos glaciarios y morfología de drumlins que dan suelos extremadamente fértiles donde se cultiva las mejores manzanas del País. De allí el nombre de "La Gran Manzana" que se atribuye a Nueva York.

Mientras todas las otras ciudades de la costa tenían el obstáculos de las Montañas Azules y los Apalaches, Nueva York consiguió incorporar fácilmente el interland del Este, compitiendo en mejores condiciones con Chicago, como puerto de ultramar.

Otro factor muy importante a tener en cuenta es el descubrimiento de yacimientos de petróleo en Pennsylvania, cuando la industrialización comenzó a depender menos del carbón y más del petróleo

Poco a poco se fue generando la gran región industrial situada entre está última ciudad y Nueva York, en tanto que las ciudades del este se expandieron hasta originar una gigantesca megalópolis que sigue la dirección de la línea de falla y se conoce como Waston, desde Washington a Boston (Fig. 7).

Así cono hemos visto la influencia de la Geomorfología en la organización del espacio es posible, para finalizar ver como los factores culturales pueden sobreimponerse a ese condicionamiento.

Un ejemplo que podemos mencionar en la zona considerada es el valle del río San Lorenzo, ocupado por el norte por la colonización francesa y al sur por la inglesa, cuyo estilo de organización es muy diferente.

En el área francesa los predios se distribuyen en fajas perpendiculares al camino con una organización típicamente lineal en tanto que en la inglesa la población tiende a concentrarse en burgos, de manera que aparecen una serie de núcleos urbanos a lo largo de los caminos y un parcelamiento radial (Fig. 8).

Nos ha parecido interesante presentar estos ejemplo para recalcar la indivisibilidad de la Geografía, que no es física y humana, sino la interacción de ambos aspectos a lo largo del tiempo que origina la organización espacial y por lo tanto que la historia es muy importante en cuanto explica los procesos y factores que influyeron sobre el paisaje a lo largo del tiempo, la forma en que el hombre se adaptó a los condicionantes naturales y las modificaciones que introdujo en aquél.

\section{Bibliografia.}

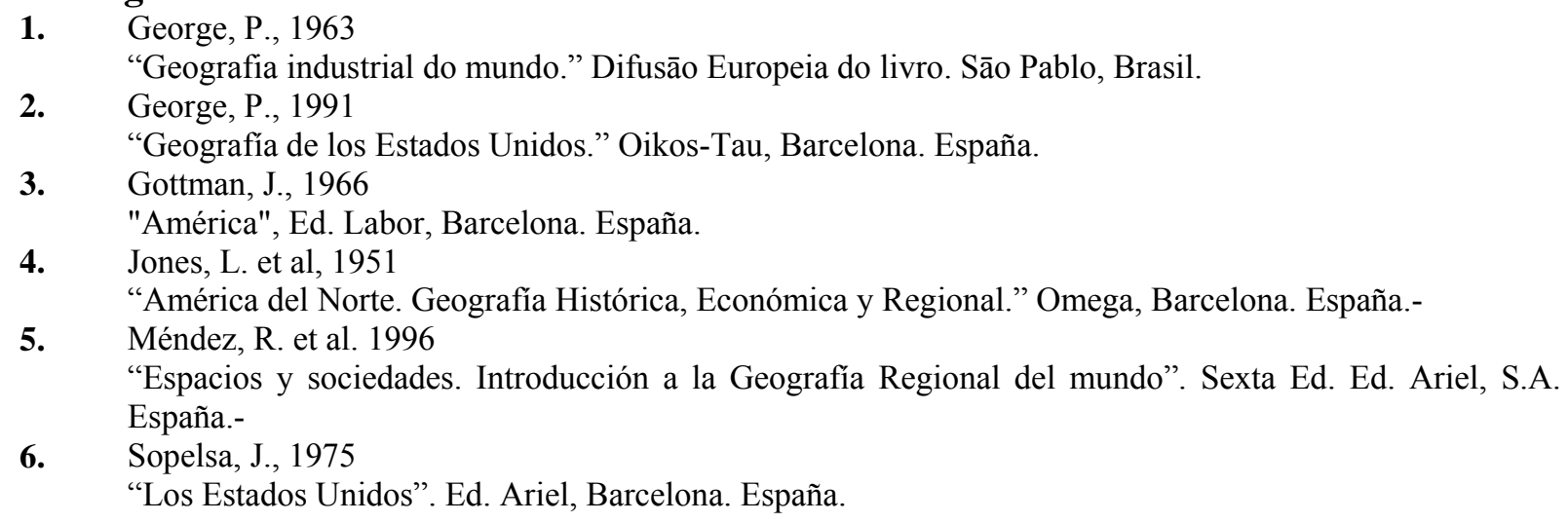

